\title{
Curcumin mediated suppression of nuclear factor-KB promotes chondrogenic differentiation of mesenchymal stem cells in a high-density co-culture microenvironment
}

\author{
Constanze Buhrmann¹, Ali Mobasheri², Ulrike Matis ${ }^{3}$ and Mehdi Shakibaei*1
}

\begin{abstract}
Introduction: Osteoarthritis (OA) and rheumatoid arthritis (RA) are characterised by joint inflammation and cartilage degradation. Although mesenchymal stem cell (MSC)-like progenitors are resident in the superficial zone of articular cartilage, damaged tissue does not possess the capacity for regeneration. The high levels of pro-inflammatory cytokines present in OA/RA joints may impede the chondrogenic differentiation of these progenitors. Interleukin (IL)$1 \beta$ activates the transcription factor nuclear factor-KB (NF-KB), which in turn activates proteins involved in matrix degradation, inflammation and apoptosis. Curcumin is a phytochemical capable of inhibiting IL-1 $\beta$-induced activation of NF-KB and expression of apoptotic and pro-inflammatory genes in chondrocytes. Therefore, the aim of the present study was to evaluate the influence of curcumin on IL-1 $\beta$-induced NF-KB signalling pathway in MSCs during chondrogenic differentiation.
\end{abstract}

Methods: MSCs were either cultured in a ratio of 1:1 with primary chondrocytes in high-density culture or cultured alone in monolayer with/without curcumin and/or IL-1 $\beta$.

Results: We demonstrate that although curcumin alone does not have chondrogenic effects on MSCS, it inhibits IL-1 $\beta$ induced activation of NF-KB, activation of caspase-3 and cyclooxygenase-2 in MSCs time and concentration dependently, as it does in chondrocytes. In IL-1 $\beta$ stimulated co-cultures, four-hour pre-treatment with curcumin significantly enhanced the production of collagen type II, cartilage specific proteoglycans (CSPGs), $\beta 1$-integrin, as well as activating MAPKinase signaling and suppressing caspase-3 and cyclooxygenase-2.

Conclusions: Curcumin treatment may help establish a microenvironment in which the effects of pro-inflammatory cytokines are antagonized, thus facilitating chondrogenesis of MSC-like progenitor cells in vivo. This strategy may support the regeneration of articular cartilage.

\section{Introduction}

Osteoarthritis (OA) and rheumatoid arthritis (RA) involve degenerative changes in the joint, leading to loss of function, pain and significant disability [1]. OA and RA are not only common joint diseases in the elderly population but increasingly they affect young individuals. Collectively, they represent a large proportion of orthopaedic cases [2]. Articular cartilage is an avascular, alymphatic and aneural tissue with bradytrophic characteristics and a

* Correspondence: mehdi.shakibaei@med.uni-muenchen.de

${ }^{1}$ Musculoskeletal Research Group, Institute of Anatomy, Ludwig-MaximiliansUniversity Munich, Pettenkoferstrasse 11, D-80336 Munich, Germany Full list of author information is available at the end of the article very poor capacity for self-repair and regeneration [3]. Cartilage repair is ineffective and often leads to replacement of the articular cartilage by a mechanically inferior fibrocartilage tissue thus promoting progressive degeneration and impairment of joint function [4]. This inherent weakness in cartilage repair highlights the acute need for novel treatments using tissue engineering and regenerative medicine, and innovative new regenerative strategies that involve stimulation of articular cartilage repair in vivo.

$\mathrm{OA}$ is characterized by an imbalance between cartilage anabolism and catabolism. The local production and 
release of pro-inflammatory cytokines (interleukin-1 $\beta$ (IL-1 $\beta$ ), interleukin-6 (IL-6), tumor necrosis factor- $\alpha$ (TNF- $\alpha)$ ) play a central role in the pathogenesis of OA [57]. It is well known that IL- $1 \beta$ and TNF- $\alpha$ production activates the transcription factor nuclear factor- $\mathrm{KB}$ (NF$\kappa B)$ in chondrocytes. Once activated, NF- $\mathrm{kB}$ translocates into the nucleus, where it induces the expression of distinct subsets of genes encoding inflammatory, apoptotic and extracellular matrix (ECM) degrading enzymes. NF$\mathrm{\kappa B}$ activates the expression of matrix degrading enzymes such as matrix metalloproteinases (MMPs) and enzymes responsible for production of prostaglandins (that is, cyclooxygenase-2 (COX-2)) leading to enhanced degradation of the ECM and induction of pain [8]. Additionally, in articular chondrocytes, NF- $\mathrm{kB}$ stimulates the production of pro-inflammatory catabolic cytokines, which induce apoptosis through activation of the proapoptotic enzyme caspase- 3 and cleavage of the DNA repair enzyme poly(ADP-ribose)polymerase (PARP) [9].

During embryonic development, cartilage develops from mesenchymal stem cells (MSCs) by condensation and differentiation. Recent studies have shown that MSClike progenitors also exist in the superficial zone of articular cartilage and that their abundance in arthritic cartilage is elevated [10]. Despite this, cartilage regeneration in vivo is inefficient and the resulting fibrocartilage is structurally and functionally inadequate. A possible explanation for this lack of regeneration is that the ongoing inflammatory processes that occur during the course of OA/RA result in higher synovial and circulating levels of pro-inflammatory cytokines, which may in turn impede the chondrogenic differentiation of cartilage resident progenitors. Therefore, blocking the pro-inflammatory cytokine induced cartilage degeneration and inflammatory cascades might create a more suitable microenvironment for the chondrogenesis of MSC-like progenitors.

In recent years the phytochemical curcumin has been identified as a potent anti-inflammatory substance in several diseases such as cancer, inflammatory bowel disease, pancreatitis, chronic anterior uveitis and arthritis [9,1116]. Curcumin is a natural yellow orange dye derived from the rhizome of Curcuma longa. It is insoluble in water but is soluble in ethanol, dimethylsulfoxide and other organic solvents. It has a melting point of $183^{\circ} \mathrm{C}$ and a molecular weight of 368.37 . Commercial curcumin contains three major components: Diferuloylmethane $(82 \%)$ and its derivatives demethoxycurcumin (15\%) and bisdemethoxycurcumin (3\%), together referred to as curcuminoids [9,11-16], all of which have anti-inflammatory activity. Curcumin reduces tumor cell survival, tumor expansion and secondary inflammation via NF- $\mathrm{kB}$ inhibition [13,17]. Further, it suppresses constitutive IкB $\alpha$ phosphorylation through the inhibition of ІкB kinase
$[13,18]$. There is increasing interest in curcumin as a therapeutic option for OA and RA, with evidence that curcumin inhibits the IL- $1 \beta$-induced activation of NF- $\mathrm{KB}$ in human articular chondrocytes $[9,14]$. Furthermore, in a recent study we have demonstrated that curcumin exerts anti-apoptotic effects on IL-1 $\beta$ stimulated human chondrocytes through inhibition of caspase- 3 activation and PARP cleavage [15].

The aim of the present investigation was to evaluate whether IL-1 $\beta$ stimulated MSCs (either alone or in a coculture model of OA with primary chondrocytes) pretreated with curcumin may impede the adverse effects of this pro-inflammatory cytokine and create a more suitable microenvironment for the chondrogenic differentiation of cartilage resident progenitor cells.

\section{Materials and methods \\ Antibodies and reagents}

Polyclonal anti-collagen type II antibody (PAB746), monoclonal anti-adult cartilage-specific proteoglycan antibody (MAB2015), anti- $\beta 1$-integrin antibody (MAB1965), and alkaline phosphatase linked sheep antimouse (AP303A) and sheep anti-rabbit secondary antibodies (AP304A) for immunoblotting and immuno-electron labelling were purchased from Chemicon International, Inc. (Temecula, CA, USA). Monoclonal anti- $\beta$-Actin (A4700) was purchased from Sigma, St. Louis, MO, USA). Monoclonal anti-Sox-9 was purchased from Acris Antibodies GmbH, Hiddenhausen, Germany. Monoclonal anti-phospho-p42/p44 ERK1/2 antibody (610032) and polyclonal anti-Shc antibody (610082) were purchased from BD (BD Biosciences, Erembodegem, Belgium). Polyclonal anti-active caspase-3 (AF835) was purchased from R\&D Systems (Abingdon, UK). Antibodies against phospho-specific IкB $\alpha$ (Ser 32/36) and against anti-phospho-specific p65(NF- $\mathrm{kB}) /($ Ser536) were obtained from Cell Technology (Beverly, MA, USA). Curcumin with a purity $>95 \%$ was purchased from Indsaff (Punjab, India). This commercial source of curcumin contains three major components: Diferuloylmethane (the most abundant and active component of turmeric) $(82 \%)$ and its derivatives demethoxycurcumin $(15 \%)$ and bisdemethoxycurcumin (3\%), together referred to as curcuminoids $[9,11-16]$. Curcumin was dissolved in dimethylsulfoxide (DMSO) as a stock concentration of $500 \mu \mathrm{M}$ and stored at $-80^{\circ} \mathrm{C}$. Serial dilutions were prepared in culture medium.

\section{Cell culture}

Mesenchymal stem cells (MSCs) were isolated from canine adipose tissue biopsies and primary canine chondrocytes were isolated from cartilage from the femoral head. Samples were obtained during total hip replacement surgery with fully-informed owner consent and eth- 
ical project approval from the ethical review committee of the Ludwig-Maximilians-University, Munich, Germany. Chondrocytes and MSCs used in co-culture experiments were always from the same animal. In total, the experiments were performed three times and samples from three different donors were used. Donor ages ranged from five to seven years.

Briefly, for MSCs isolation, adipose tissue was cut into small pieces and digested with collagenase $0.2 \%$ in Ham'sF12 in a water bath at $37^{\circ} \mathrm{C}$ for two hours. Digested adipose tissue was centrifuged at $1,000 \mathrm{~g}$ for five minutes and the pellet was resuspended in cell culture medium consisting of DMEM/Ham's-F12 1:1, 10\% FCS, 1\% partricin solution, $1 \%$ penicillin/streptomycin solution (10 000 $\mathrm{IU} / 10000 \mathrm{IU}$ ), $75 \mu \mathrm{g} / \mathrm{ml}$ ascorbic acid, $1 \%$ essential amino acids and 1\% Glutamine, all obtained from Seromed (Munich, Germany) in a T75 cell culture flask and incubated at $37^{\circ} \mathrm{C} / 5 \% \mathrm{CO}_{2}, 95 \%$ humidity. After four days, non-adherent cells were discarded by washing with Hank's salt solution. The medium was changed three times per week. Adherent cells were split following formation of fibroblast-like cell colonies and upon reaching 60 to $70 \%$ confluence, and were sub-cultured until the third or fourth passage was achieved. As there are no definitive MSC specific cellular markers, we identified them by their ability to adhere to tissue culture plastic in vitro, through their multilineage differentiation potential in vitro and through a combination of expression and lack of defined markers (CD105+, CD90+, CD45-, CD34-) [1922].

For chondrocyte isolation the cartilage sample was sliced into 1 to $2 \mathrm{~mm}$ thick slices and incubated first with pronase (2\%/Hams-F12) (Roche Diagnostics, Mannheim, Germany), followed by collagenase incubation $(0.2 \% /$ Ham's-F12) (Sigma) in a shaking water bath at $37^{\circ} \mathrm{C}$. The digested sample was centrifuged at $1,000 \mathrm{~g}$ for five minutes and cells plated at $1 \times 10^{6}$ cells per T75 flask at $37^{\circ} \mathrm{C} /$ $5 \% \mathrm{CO}_{2}$. The first medium change was performed after 24 hours, and the following medium changes three times per week. Chondrocytes were split at approximately $70 \%$ confluency and passaged twice.

\section{High-density culture}

Three-dimensional high-density cultures at the air-liquid interface were prepared as previously described [23]. Cells were centrifuged at $1,000 \mathrm{~g}$ for five minutes and around one million cells (approximately $8 \mu \mathrm{l}$ ) from the cell pellet were pipetted directly onto a nitrocellulose filter on a steel grid. This model allows the cells to aggregate, forming a distinct pellet, which was examined after 14 days.

High-density culture pellets either consisted only of MSCs or primary chondrocytes, or a mixture of MSCs and primary chondrocytes (1:1) (co-culture). In all exper- iments, cultures and co-cultures were either incubated with cell culture medium (10\% FCS) or with a chondrogenic induction medium as described by Pittenger et al. [24] consisting of DMEM base medium, D-(+)-glucose $0.35 \mathrm{~g} / 100 \mathrm{ml}$ (Sigma, Cat No. G7021), ITS +1 liquid media supplement $(10 \mu \mathrm{g} / \mathrm{ml}$ insulin, $5.5 \mu \mathrm{g} / \mathrm{ml}$ transferrin, $5 \mathrm{ng} / \mathrm{ml}$ selenium, $0.5 \mathrm{mg} / \mathrm{ml}$ bovine albumin, $4.7 \mu \mathrm{g} /$ $\mathrm{ml}$ linoleic acid (Sigma, Cat. No. I-2521), $0.1 \mathrm{mM}$ ascorbate-2-phosphate (Sigma, Cat. No. A-8960), 10-7 M dexamethasone (Sigma, Cat. No. D-8893), penicillin/ streptomycin solution (10,000 IU/10,000 IU/100 ml). 10 ng/ml hTGF $\beta 1$ (Acris Antibodies GmbH, Hiddenhausen, Germany) were added fresh to the medium before each medium change. Furthermore, some cultures and co-cultures were then incubated with one of the following treatments: curcumin only; pre-stimulated with curcumin for four hours in suspension and then transferred to high-

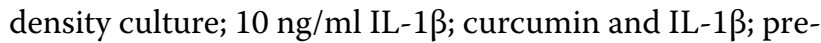
stimulated with curcumin for four hours in suspension and then brought into high-density culture and stimulated with IL-1 $\beta$; or pre-stimulated with curcumin for four hours in suspension and then brought into high-density culture and stimulated with IL-1 $\beta$ and curcumin. Medium changes were made every three days.

\section{Time and concentration dependent experiments in monolayer culture}

To examine in more detail the interaction between curcumin and IL- $1 \beta$ in MSCs and the pathological pathways involved, monolayer cultures of MSCs were evaluated. First, MSCs were cultured with various concentrations of curcumin $(0,0.5,1,2$ and $5 \mu \mathrm{M})$ for four hours, followed by 24 hours $10 \mathrm{ng} / \mathrm{ml}$ IL- $1 \beta$ stimulation. Second, MSC cultures were pre-treated for four hours with $5 \mu \mathrm{M}$ curcumin followed by one hour $10 \mathrm{ng} / \mathrm{ml} \mathrm{IL-1} \beta$ stimulation. Whole cell lysates, cytoplasmic extracts and nuclear extracts were taken at various time points and evaluated with Western blotting.

\section{Electron microscopy}

Transmission electron microscopy was performed as previously described [25]. High-density co-cultures were fixed for one hour in Karnovsky-fixative fixative and post-fixed in $1 \% \mathrm{OsO}_{4}$ solution. After dehydration, pellets were embedded in Epon, ultrathin cuts were made on a Reichert-Ultracut $\mathrm{E}$ and contrasted with a mixture of $2 \%$ uranyl acetate/lead citrate. A transmission electron microscope (TEM 10, Zeiss, Jena, Germany) was used to examine the co-cultures. To quantify apoptosis, the number of cells exhibiting typical morphological features of apoptotic cell death was determined by scoring 100 cells from 30 different microscopic fields per culture and the number of apoptotic cells expressed as an indicator of MSC culture degradation. 


\section{Western blot analysis}

For Western blotting, total cell proteins were either extracted from the cell cultures with lysis buffer on ice for 30 minutes or nuclear extracts and cytoplasmic extracts prepared as previously described [9]. Total protein content was measured with the bicinchoninic acid system (Uptima, France) using bovine serum albumin as a standard. Samples were further reduced with 2-mercaptoethanol and total protein concentrations adjusted. Proteins (500 ng per lane total protein) were separated with SDSPAGE under reducing conditions on $5 \%, 7 \%, 10 \%$ or $12 \%$ gels and then blotted onto nitrocellulose membranes using a trans blot apparatus (Bio-Rad, Munich, Germany). After blocking for two hours in 5\% (w/v) skimmed milk powder in phosphate buffered saline (PBS)/0.1\% Tween 20, membranes were incubated with the primary antibodies (overnight, $4^{\circ} \mathrm{C}$ ), followed by incubation with the alkaline phosphatase conjugated secondary antibodies for two hours at room temperature. Finally, specific antigen-antibody complexes were detected using nitroblue tetrazolium and 5-bromo-4-chloro-3indoylphosphate ( $p$-toluidine salt; Pierce, Rockford, IL, USA) as substrates for alkaline phosphatase. Specific binding was quantified by densitometry using "Quantity One" (Bio-Rad Laboratories Inc. Munich, Germany).

\section{Results}

\section{Characterisation of canine adipose tissue derived} mesenchymal stem cells

In order to demonstrate that the cells isolated from the canine adipose tissue (Figure 1A) are indeed mesenchymal stem cells (MSCs) we differentiated them to adipocytes, osteoblasts and chondrocytes (Figure 1B, C). After three weeks' treatment with the adipogenic induction medium, the cells contained abundant amounts of vacuoles and Oil Red $\mathrm{O}$ staining for fat revealed that these vacuoles contained neutral lipids (B). After three weeks culture time with the osteogenic induction medium the cells changed to a more polygonal appearance, formed nodules and were stained positive with von Kossa stain for mineral deposition (C). Alcian blue staining after 14 days in culture revealed a high content of cartilage specific proteoglycans in induced cultures (D). Additionally, the isolated MSCs showed a strong positive signal for the stem cell specific markers CD90+ and CD105+ (Figure 1E, F). In contrast to this they were clearly labelled negative for the hematopoietic stem cell markers CD45- and CD34- (Figure 1G, H).

\section{Curcumin alone does not have a chondro-inductive effect on pure MSC high-density cultures}

To study the effects of curcumin on MSCs after 14 days of cultivation in three-dimensional high-density culture, ultrastructural evaluations were performed (Figure 2A). In control cultures, MSCs did not survive, but underwent apoptosis or necrosis and mainly cell debris was observed (a). Treatment of the cultures with the chondrogenic induction medium stimulated chondrogenesis (b). The formation of cartilage nodules consisting of large rounded viable cells (containing large quantities of endoplasmic reticulum, mitochondria and other cellular organelles) embedded in a fine structured, highly organised ECM was observed. Treatment of MSCs with curcumin alone did not stimulate chondrogenesis and, as in control cultures, mainly cell debris was observed (c, e). It did not make a difference whether cultures were either
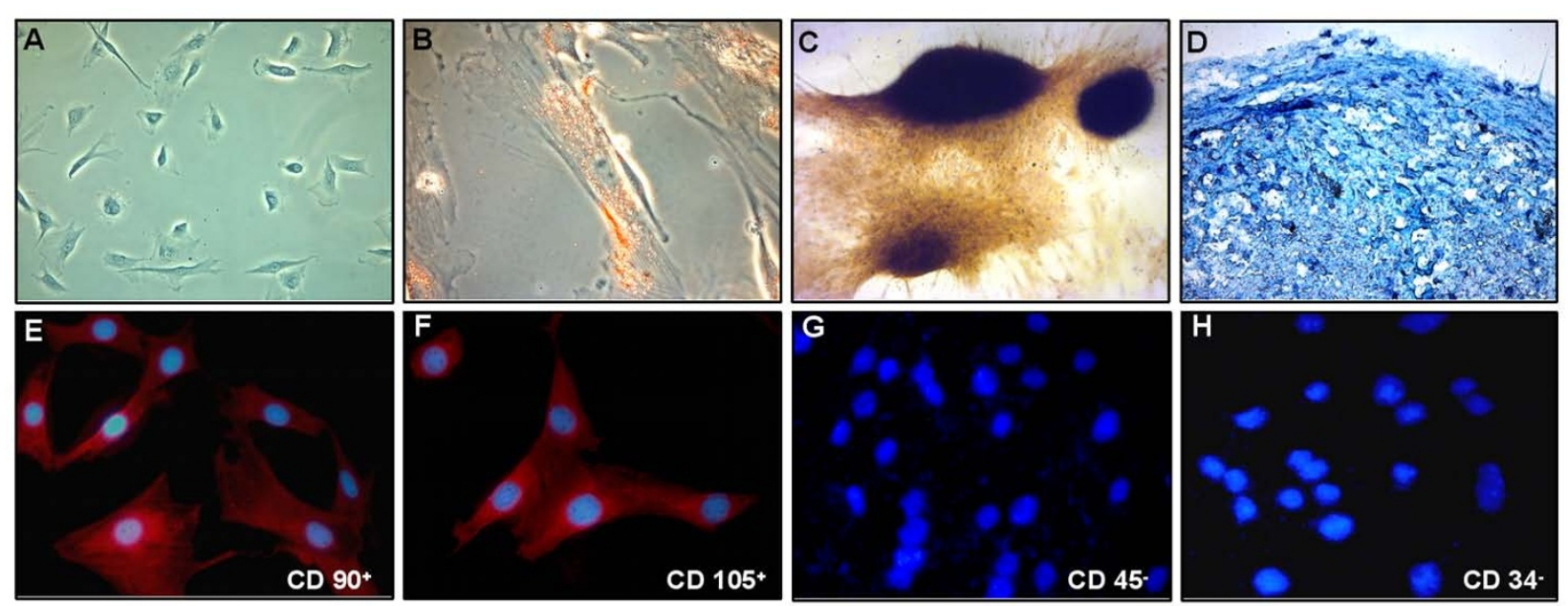

Figure 1 Characterisation of MSCs. In monolayer culture the adipose derived MSCs (A) assumed a polymorphic, fibroblast-like morphology and could be differentiated into adipocytes (B; Oil red staining), osteoblasts (C; von Kossa) and chondrocytes (D; alcian blue). The isolated MSCs showed a strong positive signal for the stem cell specific markers $\mathrm{CD}^{+}(\mathbf{E})$ and $\mathrm{CD} \mathrm{105}^{+}(\mathbf{F})$ and were negative for the hematopoietic stem cell markers CD45(G) and CD34- (H). Magnification: A: 5x; B: 40x; C: 20x; D: 20x; E-H: 40x. 


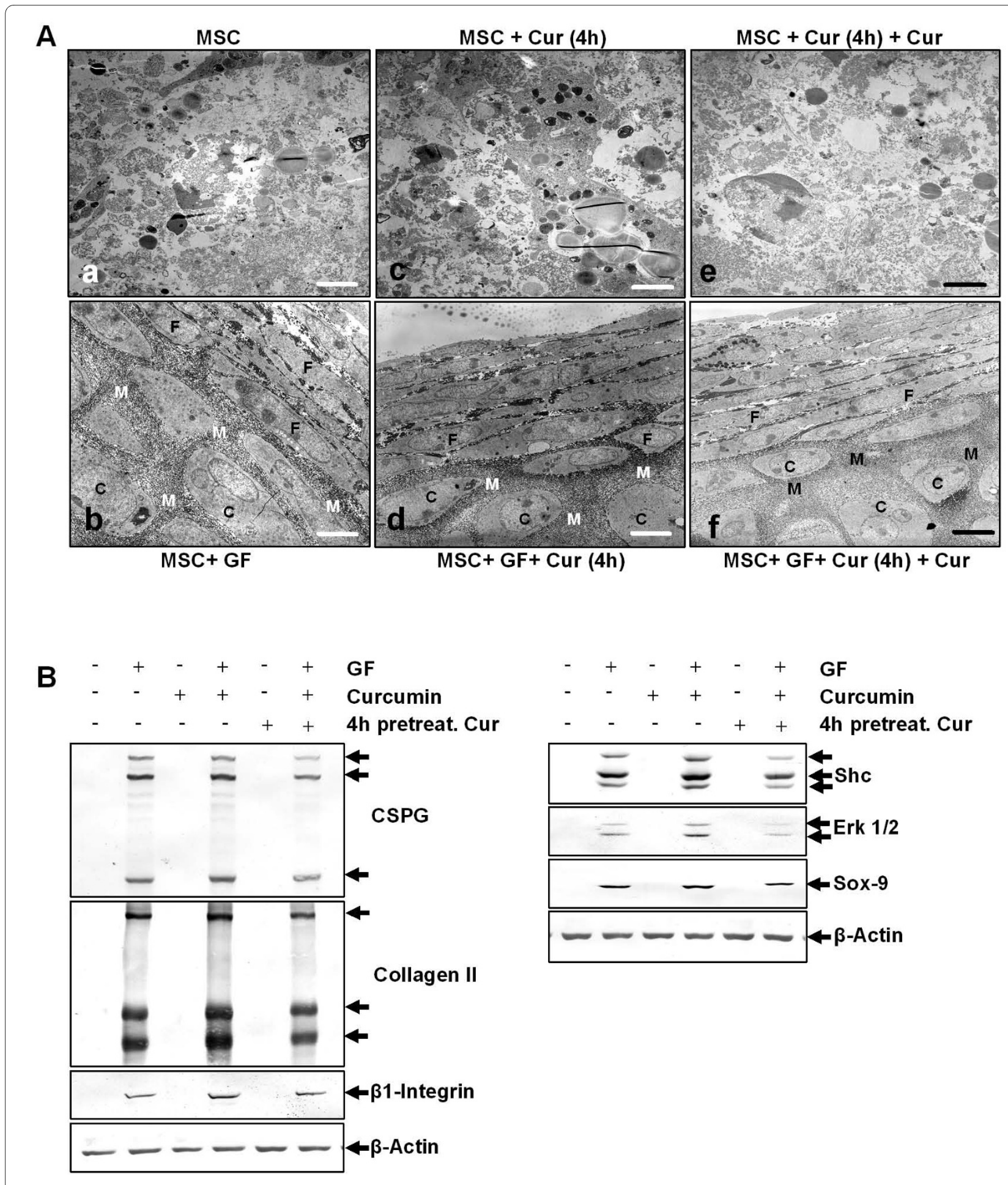

Figure 2 Curcumin alone does not enhance chondrogenesis in MSCs. A: 14 days high-density culture. Apoptosis or necrosis was observed in MSC cultures (a), MSC cultures treated with curcumin (c) or MSC cultures pre-stimulated four hours with curcumin, followed by incubation with curcumin (e). In contrast, chondrogenesis was observed in MSC cultures treated with the chondrogenic induction medium alone (b), in combination with curcumin (d) or a hour-hour pre-stimulation with curcumin, followed by a combination of curcumin and the chondrogenic induction medium (f). Magnification, 6000x; bar, 1 mm; C, chondrocytes; F, fibroblast-like cells; M, ECM; GF, chondrogenic induction medium. $\mathbf{B}$ : The ultrastructural findings above were confirmed by western blotting. Immunoblots of whole cell lysates were probed using antibodies that recognize CSPGs, collagen type II, $\beta 1$-integrin, Shc, activated-ERK1/2 and Sox-9. Each experiment was performed in triplicate. Expression of the housekeeping gene $\beta$-actin was not affected. $\mathrm{GF}$, chondrogenic induction medium. 
pre-treated for four hours with curcumin (c) or pretreated four hours with curcumin followed by treatment with curcumin over the entire culture period (e). In contrast, in cultures treated with curcumin and the chondrogenic induction medium (d, f) chondrogenesis was observed. Chondrogenesis was similar in cultures that were either pre-treated for four hours with curcumin (d) or cultures that were pre-treated four hours with curcumin followed by treatment with curcumin over the entire culture period (f).

Western blotting was performed to confirm these results (Figure 2B). Whole cell lysates were resolved by SDS-PAGE electrophoresis and blotted onto nitrocellulose. The membranes were probed with antibodies against cartilage specific proteoglycans (CSPG), collagen type II, $\beta 1$-integrins, Shc, activated extracellular regulated kinases 1 and 2 (ERK 1/2) and Sox-9.

In agreement with the ultrastructural findings, MSC cultures treated with the specific chondrogenic induction medium alone or in combination with curcumin produced high amounts of CSPGs, collagen type II and $\beta 1$ integrin. Here, four-hour curcumin pre-treatment followed by treatment with the chondrogenic induction medium was as effective in inducing the production of CSPGs, collagen type II and $\beta 1$-integrin as a four-hour curcumin pre-treatment followed by treatment with curcumin and the chondrogenic induction medium over the entire culture period. Further, in these cultures the chondrogenic signalling cascade was activated with high expression of Shc, activated ERK 1/2 and Sox-9. In contrast to this, cartilage specific matrix components and members of the chondrogenic signalling cascade, were not expressed in untreated cultures or cultures incubated only with curcumin. Again, it did not make a difference whether cultures were pre-treated for four hours with curcumin or pre-treated four hours with curcumin followed by treatment with curcumin over the entire culture period.

\section{Curcumin inhibits IL-1 $\beta$ activity in MSCs, enabling growth factor induced chondrogenesis}

It has been reported that IL- $1 \beta$ inhibits chondrocyte proliferation and induces apoptosis [26,27]. We therefore evaluated the effects of curcumin on MSCs stimulated with IL-1 $\beta$ and/or the chondrogenic induction medium.

Ultrastructural evaluation revealed that stimulation of MSCs with IL- $1 \beta$ either alone (Figure 3A-a), in combination with the chondrogenic induction medium (b) or in combination with four-hour curcumin pre-treatment (c) resulted in apoptosis. However, treatment with IL-1 $\beta$, curcumin and the chondrogenic induction medium lead to induction of chondrogenesis (d, e) with formation of cartilage nodules containing viable, rounded cells that were embedded in a cartilage specific matrix. In these cultures, a four-hour curcumin pre-treatment (d) was as effective in inhibiting IL-1 $\beta$ induced apoptosis as a fourhour curcumin pre-treatment followed by treatment with curcumin over the entire culture period (e).

Apoptosis was further quantified as described in Materials and Methods. The data shown in Figure 3B demonstrate a significantly increased number of apoptotic cells in cultures stimulated with IL- $1 \beta$ either alone, in combination with the chondrogenic induction medium or in combination with a four-hour curcumin pre-treatment. As shown at the ultrastructural level, the number of apoptotic cells significantly decreased in MSC cultures treated with IL-1 $\beta$, curcumin and the chondrogenic induction medium (Figure 3B). In these cultures the number of apoptotic cells was similar between cultures that were either pre-treated for four hours with curcumin or pre-treated four hours with curcumin followed by curcumin treatment over the entire culture period.

To support these ultrastructural findings, Western blotting was performed by probing whole cell lysates with antibodies against CSPGs, collagen type II, $\beta 1$-integrin, Shc, activated ERK 1/2 and Sox-9 (Figure 3C). Stimulation of MSC cultures with IL-1 $\beta$ either alone, in combination with the chondrogenic induction medium or with a four-hour curcumin pre-treatment did not lead to production of CSPGs, collagen type II, $\beta 1$-integrin and activation of Shc, ERK $1 / 2$ and Sox-9. In contrast, production of CSPGs, collagen type II and $\beta 1$-integrin was upregulated and Shc, activated ERK $1 / 2$ and the chondrogenic specific transcription factor Sox-9 was highly expressed in MSC cultures treated with IL-1 $\beta$, curcumin and the chondrogenic induction medium. Underlining the ultrastructural findings, it did not make a difference whether cultures were either pre-treated for four hours with curcumin or pre-treated four hours with curcumin followed by curcumin treatment over the entire culture period.

Further, to demonstrate the influence of curcumin on the induction of the apoptotic signalling cascade by IL- $1 \beta$ in MSCs, cultures were evaluated for activated caspase-3 and the marker of inflammation and prostaglandin production COX-2 (Figure 3D). Production of activated caspase- 3 and COX-2 expression was prominent in all MSC cultures stimulated with IL- $1 \beta$ alone and was blocked in all cultures treated with IL-1 $\beta$ and curcumin. Here, a four-hour pre-treatment with curcumin was as effective in inhibiting the IL- $1 \beta$ induced apoptotic signalling cascade as a four-hour pre-treatment with curcumin followed by curcumin treatment over the entire culture period.

\section{Curcumin promotes chondrogenesis in co-cultures stimulated with IL-1 $\beta$}

As demonstrated above, MSC cultures treated with a chondro-inductive medium undergo chondrogenesis 


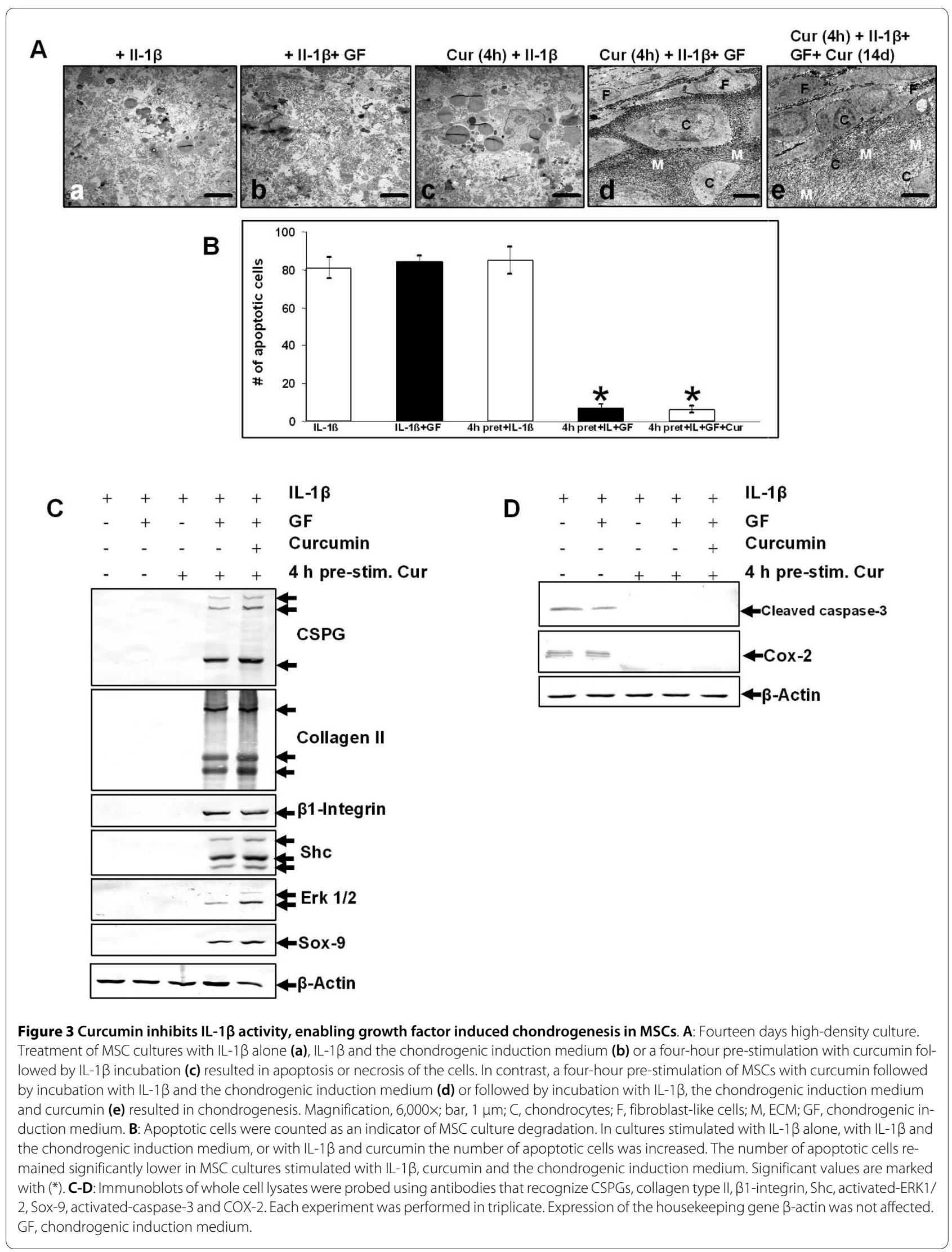


despite the presence of IL-1 $\beta$ if the apoptotic and inflammatory cascades induced by IL- $1 \beta$ are inhibited by curcumin. Next we evaluated whether the same effect can be observed in a co-culture model of MSCs and primary chondrocytes.

Ultrastructural evaluation demonstrated cellular debris in untreated MSC cultures (Figure 4A-a), and development of cartilage nodules with rounded, viable cells embedded in a highly organised, fine structured ECM in untreated primary chondrocyte cultures (b) and in untreated co-cultures (c). Stimulation of co-cultures with IL-1 $\beta$ resulted in cellular degradation (d). Curcumin treatment of the co-cultures, either as a four-hour pretreatment (e) or over the entire culture period (f) did not impede chondrogenesis. Combinational treatment of cocultures with IL-1 $\beta$ and curcumin suppressed the adverse effects of IL- $1 \beta$ on chondrogenesis $(\mathrm{g}, \mathrm{h})$. Here, inhibition of IL-1 $\beta$ induced apoptosis was as effectively blocked by a four-hour curcumin treatment $(\mathrm{g})$ as by curcumin treatment for the entire culture period (h).

Western blotting using antibodies against CSPGs, collagen type II, $\beta 1$-integrin, Shc, activated ERK $1 / 2$ and Sox-9 was performed to evaluate induction of chondrogenesis in the co-cultures on a molecular level (Figure 4B, C). Production of cartilage matrix specific markers and of chondrogenic signalling pathway members was slight in untreated MSC cultures and high in untreated primary chondrocyte cultures and untreated co-cultures (Figure $4 B, C)$. Stimulation of co-cultures with IL- $1 \beta$ alone inhibited production and expression of CSPGs, collagen type II, $\beta 1$-integrin, Shc, activated ERK $1 / 2$ and Sox-9. In contrast, co-cultures stimulated with IL-1 $\beta$ and curcumin produced high levels of chondrogenic matrix specific markers (Figure 4B) and chondrogenic signalling pathway proteins (Figure 4C). Stimulation of chondrogenesis was similar between cultures that were either pre-treated for four hours with curcumin or were pre-treated four hours with curcumin followed by curcumin treatment over the entire culture period and comparable to untreated primary chondrocyte cultures and untreated co-cultures.

To further demonstrate the influence of curcumin on the induction of the apoptotic signalling cascade by IL- $1 \beta$ in co-cultures, cultures were evaluated for activated caspase- 3 and the marker of inflammation and prostaglandin production COX-2 (Figure 4D). Activated caspase-3 and COX-2 were highly expressed in untreated MSC cultures but were not expressed in untreated primary chondrocyte cultures and untreated co-cultures. Treatment of the co-cultures with IL-1 $\beta$ alone led to high production of activated caspase- 3 and COX-2. In contrast, in co-cultures stimulated with both IL- $1 \beta$ and curcumin neither activated caspase- 3 nor COX-2 was detected. A fourhour pre-treatment of curcumin or curcumin treatment over the entire culture period both effectively inhibited IL- $1 \beta$ induced activation of caspase- 3 and COX- 2 production. These results confirm the ultrastructural findings described above and demonstrate that curcumin inhibits IL- $1 \beta$ induced apoptotic and inflammatory signalling pathways, promoting co-culture induced chondrogenesis.

\section{Curcumin suppresses IL-1 $\beta$-induced apoptotic and inflammatory responses in MSCs in a time and concentration dependent manner}

Further experiments were carried out to evaluate the interaction between curcumin and IL- $1 \beta$ in MSCs. These experiments demonstrated that curcumin suppressed IL$1 \beta$ induced activation of apoptotic and inflammatory pathways in a concentration (Figure 5) and time (Figure 6) dependent manner.

As shown in Figure 5A, treatment with as little as 0.5 $\mu \mathrm{M}$ of curcumin over the entire culture period was sufficient to significantly suppress IL- $1 \beta$ induced activation of caspase- 3 and COX- 2 production in MSCs. Pro-caspase3 production remained unaffected.

The IL-1 $\beta$-activated transcription factor nuclear factor$\kappa B(N F-\kappa B)$ plays an essential role in mediating inflammatory and apoptotic processes in chondrocytes and it is known that in chondrocytes, curcumin is able to suppress NF- $\mathrm{kB}[9,14,28]$. To evaluate whether curcumin influences IL-1 $\beta$-induced NF- $\kappa B$ in MSCs, we investigated the NF- $\mathrm{kB}$ signalling pathway. As demonstrated in Figure 5B, curcumin suppressed IL-1 $\beta$-induced activation of Iк-B $\alpha$ in MSCs. This correlated clearly with decreased NF- $\mathrm{kB}$ translocation to the nucleus. Inhibition of Ік-B $\alpha$ phosphorylation as well as NF- $\kappa B$ translocation to the nucleus was evident when curcumin was included at a concentration of $0.5 \mu \mathrm{M}$. Higher concentrations of curcumin completely blocked IL-1 $\beta$-induced activation of IK-B $\alpha$ and NF- $\kappa B$ translocation to the nucleus (Figure $5 \mathrm{~B}$ ).

Further, pre-treatment of MSC cultures with $5 \mu \mathrm{M}$ curcumin also inhibited IL- $1 \beta$-induced activation of caspase3 and COX-2 expression in a time dependent manner (Figure 6A). After 60 minutes incubation time, activation of caspase- 3 and COX-2 production was completely suppressed. Pre-treatment of MSC cultures with $5 \mu \mathrm{M}$ curcumin also inhibited activation of Iк-B $\alpha$ and NF- $\mathrm{kB}$ translocation to the nucleus in a time dependent fashion (Figure 6B). In contrast, in IL-1 $\beta$ treated control cultures, activated caspase- 3 , higher expression of COX-2, activated Iк-B $\alpha$ and higher concentrations of NF- $\mathrm{KB}$ in the nucleus were observed.

\section{Discussion}

The aim of this study was to evaluate whether curcumin has the capacity to modulate inflammatory processes in 
A

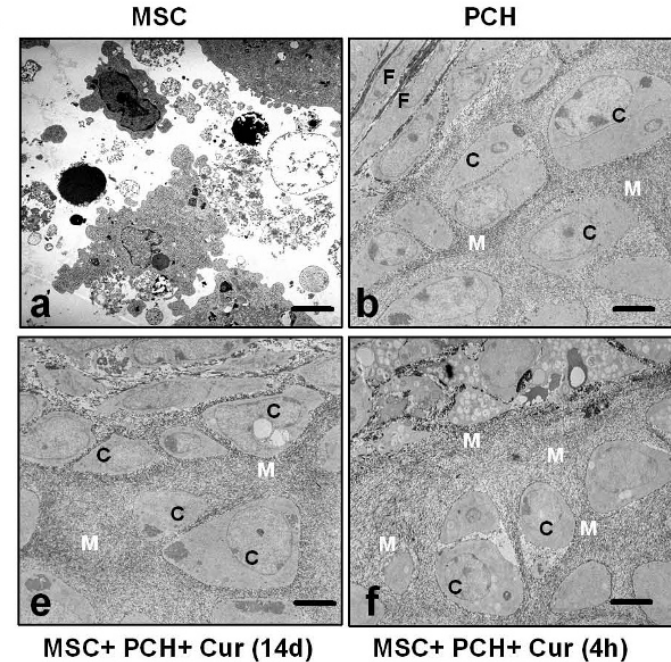

B
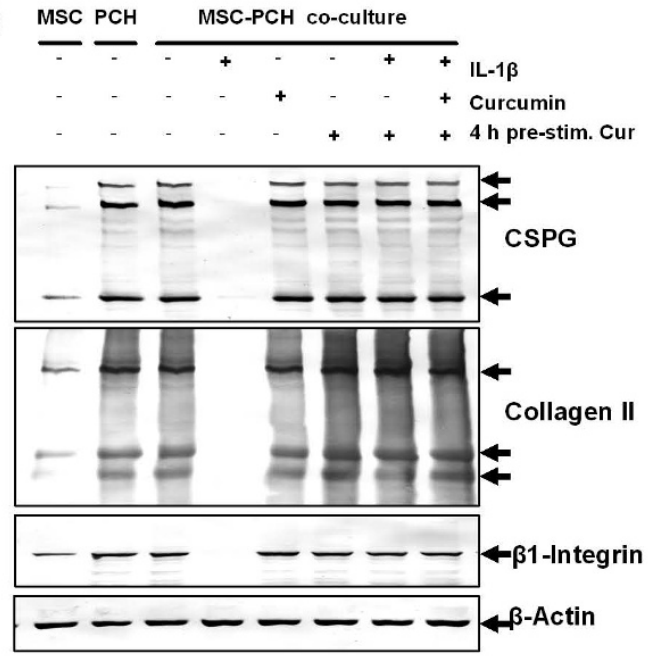

$\mathrm{MSC}+\mathrm{PCH}$
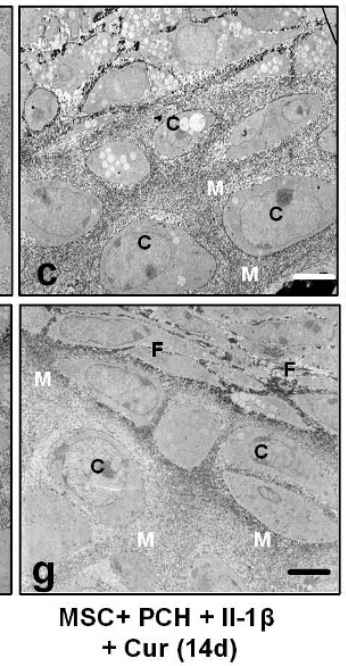

C
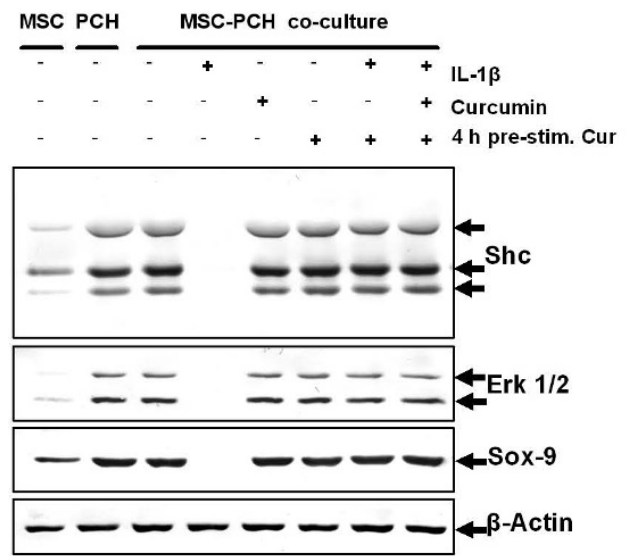

D MSC PCH MSC-PCH co-culture
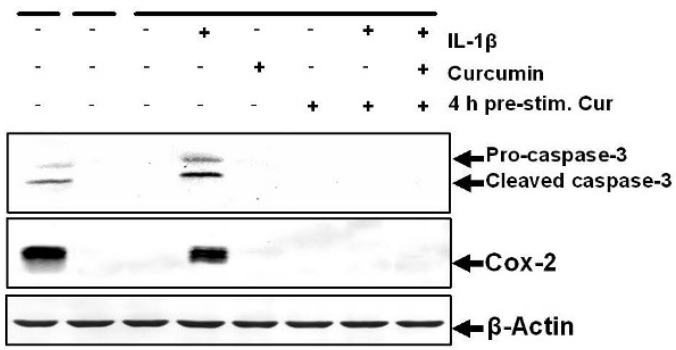

Figure 4 Curcumin inhibits IL-1 $\beta$ activity, enabling co-culture induced chondrogenesis in MSCs. A: Fourteen days high-density culture. Untreated MSC cultures became apoptotic (a). In primary chondrocyte cultures (b), co-cultures (c), co-cultures treated with curcumin (e) or co-cultures pre-stimulated four hours with curcumin (f), prominent chondrogenesis was observed. Stimulation of the co-culture with IL-1 $\beta$ alone resulted in degeneration of the cell culture (d). In contrast, a four-hour pre-stimulation of the co-culture with curcumin followed by IL-1 $\beta$ incubation ( $\mathbf{g}$ ) or a fourhour pre-stimulation of the co-culture with curcumin followed by IL-1 $\beta$ and curcumin incubation (h) inhibited the adverse effects of IL-1 $\beta$ on the chondrogenic potential of the co-culture and prominent chondrogenesis was observed. Magnification, 6,000x; bar, $1 \mu \mathrm{m} ; \mathrm{C}$, chondrocytes, F, fibroblast-like cells; M, ECM. B-D: Immunoblots of whole cell lysates were probed with antibodies against CSPGs, collagen type II, $\beta 1$-integrin, Shc, activated-ERK1/2, Sox-9, activated-caspase-3 and COX-2. In co-cultures pre-stimulated for four hours with curcumin followed either by incubation with IL$1 \beta$ alone or incubation with IL-1 $\beta$ and curcumin, prominent production of chondrogenic matrix and adhesion molecules (B), activation of the chondrogenic signalling pathway $(C)$ and down-regulation of apoptotic and inflammatory markers (D) was observed. Each experiment was performed in triplicate. Expression of the housekeeping gene $\beta$-actin was not affected. 


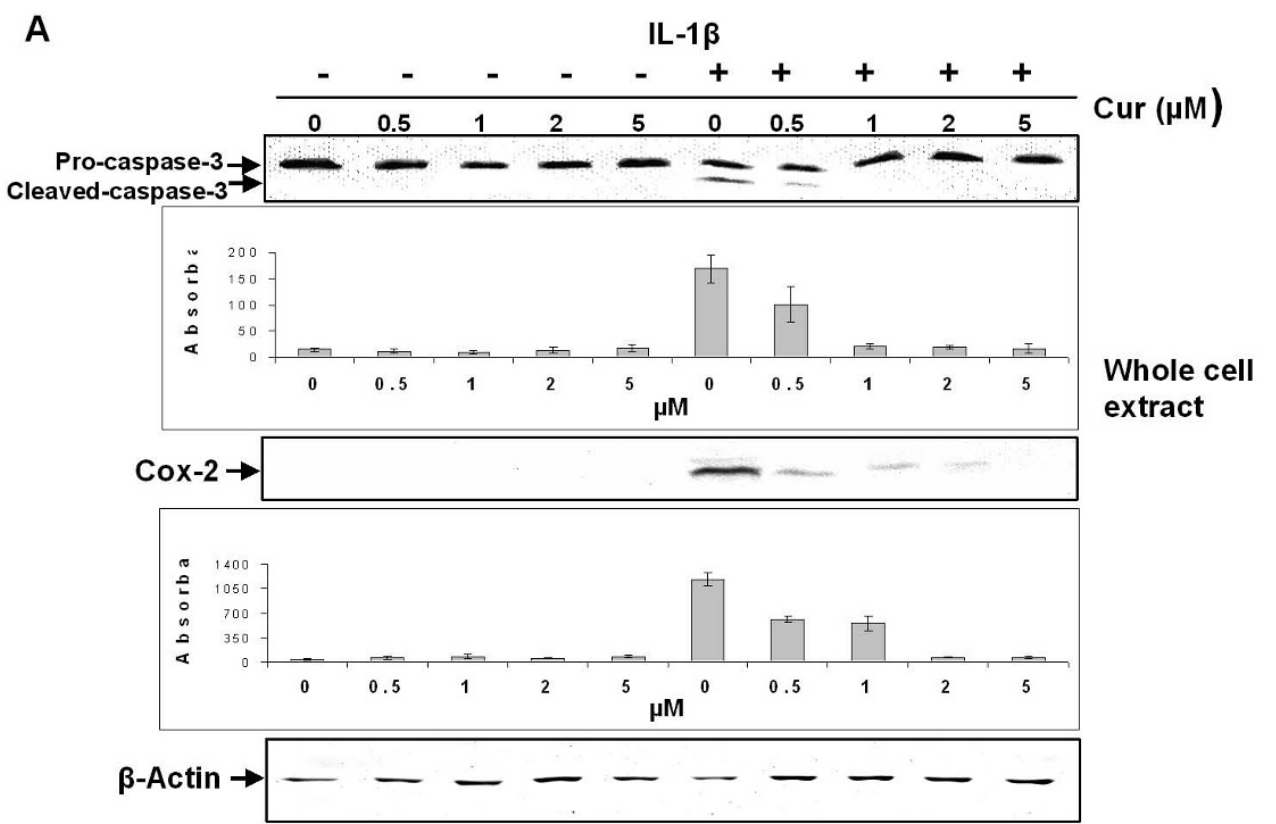

B

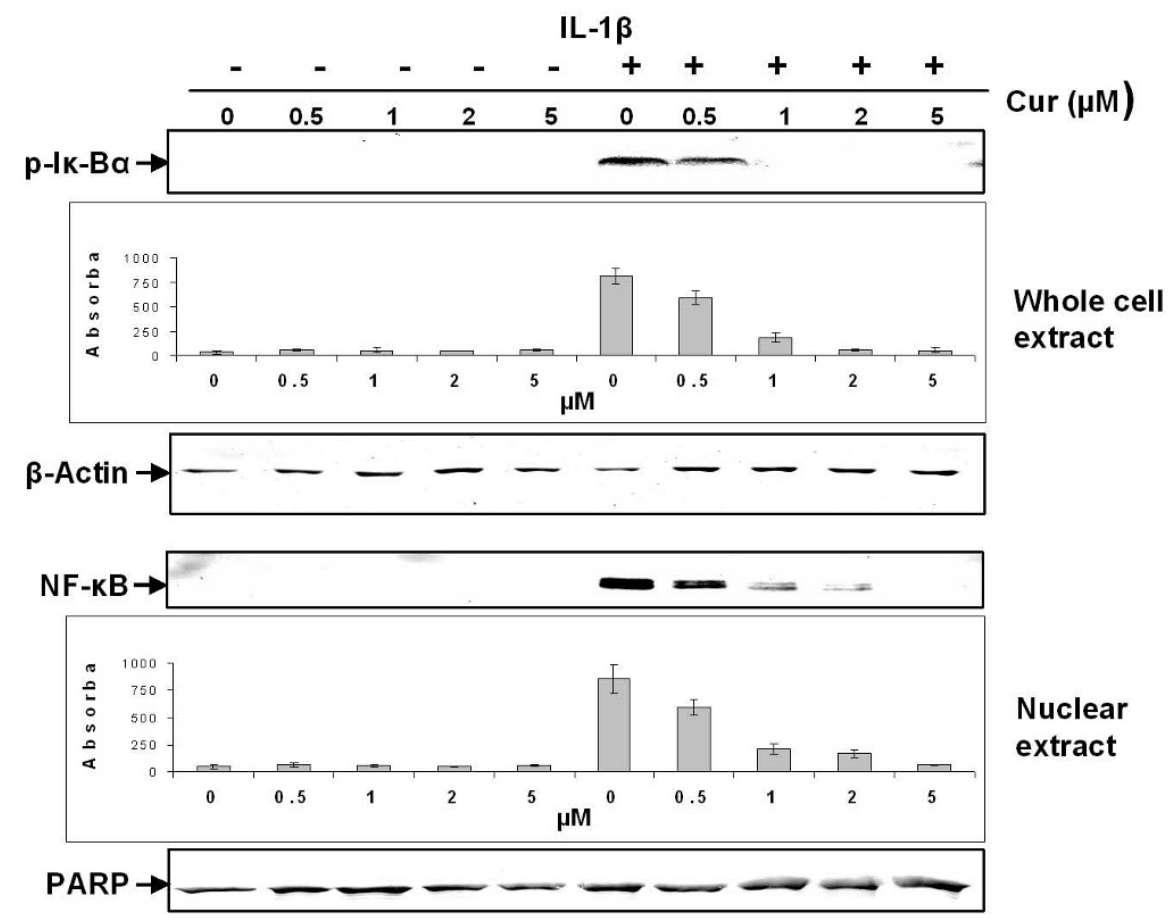

Figure 5 Curcumin suppresses IL-1 $\beta$-induced apoptotic and inflammatory responses in monolayers of MSCs in a concentration dependent manner. Monolayer cultures of MSCs were pre-stimulated for four hours with various concentrations of curcumin $(0,0.5,1,2$ and $5 \mu \mathrm{M})$ followed by $24 \mathrm{~h}$ incubation with IL-1 $\beta$, and Western blotting was performed using whole cell lysates and nuclear extracts. A: A strong dose dependent effect on IL-1 $\beta$ induced activation of caspase- 3 and COX-2 was observed. Curcumin concentrations as low as $0.5 \mu \mathrm{M}$ suppresses IL-1 $\beta$ induced activation of caspase-3 and COX-2. Higher concentrations of curcumin completely inhibited IL-1 $\beta$ induced activation of caspase-3 and production of COX-2. This was confirmed by quantitative densitometry. The mean values and standard deviations from three independent experiments are shown. Expression of the housekeeping gene $\beta$-actin was not affected. B: Curcumin exerts a strong dose dependent effect on IL-1 $\beta$ activated IK-Ba in MSCs, by suppressing phosphorylation of $\mathrm{K}-\mathrm{Ba}$ (which is already fairly robust) and NF-KB nuclear translocation at $0.5 \mu \mathrm{M}$ curcumin. Higher concentrations of curcumin blocked IL-1 $\beta$-induced activation of $\mathrm{I}$ - $\mathrm{Ba}$ and NF-KB translocation to the nucleus completely. This was confirmed by quantitative densitometry. The mean values and standard deviations from three independent experiments are shown. Expression of the housekeeping gene $\beta$-actin and the DNA repair enzyme PARP were not affected. 


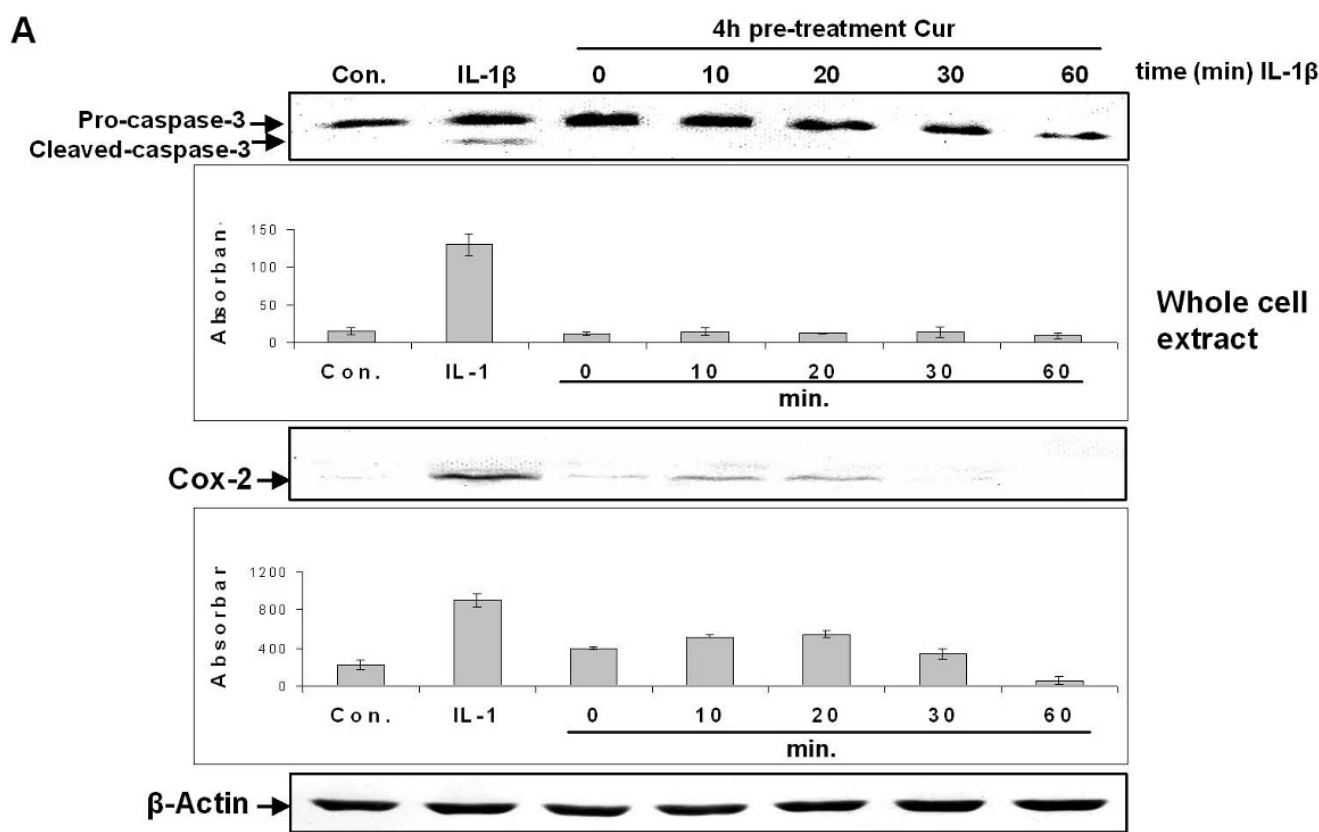

B

4h pre-treatment Cur

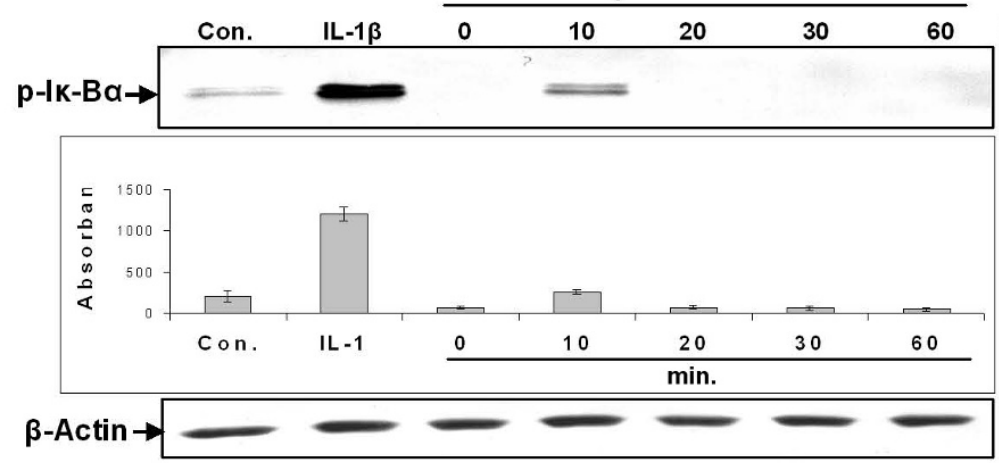

time $(\min )$ IL-1 $\beta$

\section{Cytoplasma extract}
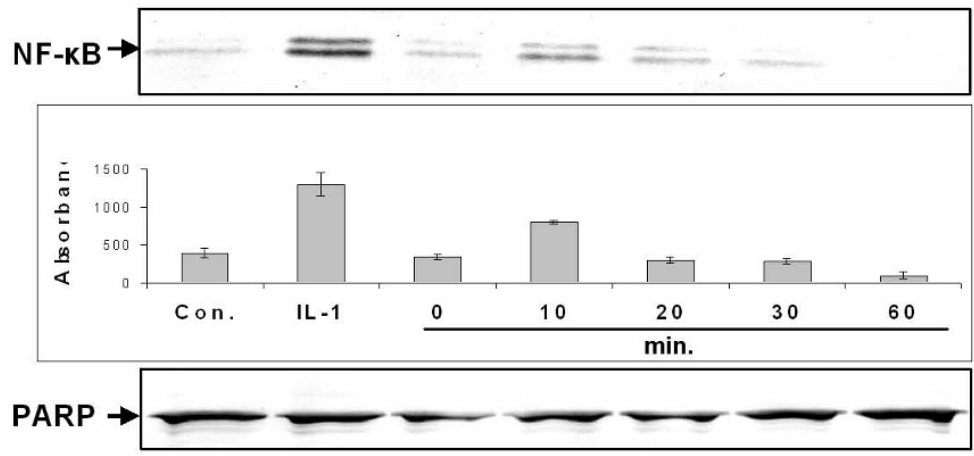

Nuclear

extract

Figure 6 Curcumin revokes IL-1 $\beta$-induced apoptotic and inflammatory responses in monolayers of MSCs in a time dependent manner. MSC Monolayer cultures were pre-stimulated for four hours with $5 \mu \mathrm{M}$ curcumin followed by $1 \mathrm{~h}$ stimulation with IL-1 $\beta$. Whole cell lysates, cytoplasmic and nuclear extracts were evaluated by western blotting at various time points. A: A four-hour pre-stimulation with $5 \mu M$ curcumin suppressed IL-1 $\beta$ induced activation of caspase-3 and expression of COX-2 in a time dependent manner. This was confirmed by quantitative densitometry. The mean values and standard deviations from three independent experiments are shown. Expression of the housekeeping gene $\beta$-actin was not affected. $\mathbf{B}$ Western blotting against activated $\mathrm{I}_{\mathrm{K}-\mathrm{B} a}$ and NF-KB demonstrated that in cultures pre-stimulated with $5 \mu \mathrm{M}$ curcumin for four hours, followed by onehour IL-1 $\beta$ stimulation, neither activation of I $\mathrm{K}-\mathrm{Ba}$ nor translocation of NF-KB to the nucleus can be demonstrated. This was confirmed by quantitative densitometry. The mean values and standard deviations from three independent experiments are shown. Expression of the housekeeping gene $\beta$ actin and the DNA repair enzyme PARP were not affected. 
MSCs and thus support chondrogenesis in an in vitro model of OA incorporating MSCs, primary chondrocytes and pro-inflammatory cytokines.

Our observations lead to the following conclusions: (1) Curcumin itself does not have any chondro-inductive potential in MSCs, and treatment of MSCs with IL-1 $\beta$ leads to cell apoptosis. (2) Although co-treatment of MSCs with curcumin and IL- $1 \beta$ does not promote chondrogenesis, it clearly inhibits up-regulation of pro-inflammatory and apoptotic signalling cascades in MSCs; (3) If MSCs receive a chondrogenic stimulus, curcumin mediated inhibition of IL-1 $\beta$-induced catabolic signalling cascades enables chondrogenic differentiation. (4) This effect is observed either by pre-treatment with curcumin (four hours) or curcumin incubation over the entire culture period. (5) Chondrogenic stimulation can be achieved either with a chondro-inductive medium or through direct, close contact co-culture of MSCs with primary chondrocytes; (6) Similar to chondrocytes, curcumin in MSCs targets the Iк-B $\alpha$ cascade, inhibiting IL$1 \beta$-induced Ік-B $\alpha$ phosphorylation and NF-кB nuclear translocation; (7) The effects of curcumin on the IL-1 $\beta$ signalling pathway in MSCs are time and concentration dependent.

OA and RA are characterised by high levels of proinflammatory cytokines in the articular joint. These are produced by synovial lining cells, macrophages and the chondrocytes themselves further exacerbating cartilage degrading and degenerative processes [29,30]. Although, as in numerous other tissues, MSC-like progenitors are also resident in adult cartilage tissue [10], these degrading and degenerative processes gradually lead to an imbalance between cartilage catabolism and anabolism, impeding MSC chondrogenesis. It has been reported that activation of NF- $\mathrm{KB}$ is the key to induction of inflammation during $\mathrm{OA}$ and $\mathrm{RA}$, and that NF- $\mathrm{kB}$ inhibition might prove to be a potential concept for arthritis treatment $[31,32]$.

The polyphenol curcumin, derived from the rhizomes of Curcuma longa is a promising therapeutic agent for the treatment of OA and RA as it has pro-apoptotic properties in synovial lining cells $[33,34]$ and has been shown to have anti-inflammatory and anti-apoptotic effects in chondrocytes $[14,15]$. In chondrocytes these effects are mainly mediated by inhibiting IL- $1 \beta$-induced activation of NF- $\mathrm{kB}$ and thus suppression of caspase- 3 activation, of production of COX-2 and upregulation of MMPs [9].

In this study we demonstrate that the IL- $1 \beta$-induced catabolic signalling cascade is suppressed by curcumin in MSCs as well as in chondrocytes. Further, we clearly demonstrate that IL- $1 \beta$ induced Ik-B $\alpha$ activation is suppressed by curcumin, resulting in suppression of NF- $\mathrm{kB}$ translocation to the nucleus and attenuated activation of caspase- 3 and COX-2 production. Taken together, our experiments on MSCs demonstrate that time and concentration dependent effects of curcumin inhibit the induction of degradative and inflammatory pathways by IL-1 $\beta$ through revoking activation of caspase-3, production of COX-2, phosphorylation of IK-B $\alpha$ and inhibition of nuclear translocation of NF- $\mathrm{kB}$.

Interestingly, although we demonstrated that MSCs in high-density culture cannot survive without a chondrogenic stimulus, stimulating these untreated cultures with IL- $1 \beta$ and curcumin neither activated caspase- 3 nor production of COX-2. This suggests that although MSCs alone in this high-density model do not survive without a necessary stimulus, they become necrotic rather than apoptotic.

We did not observe a chondrogenic effect of curcumin alone on MSCs, despite demonstrating anti-inflammatory and anti-apoptotic effects of curcumin in MSCs. However, given the necessary stimulus, chondrogenesis was observed. This demonstrates that curcumin interferes with the IL-1 $\beta$ induced apoptotic pathways in MSCs thus providing a suitable microenvironment allowing MSCs to undergo chondrogenesis even in the presence of IL-1 $\beta$, as long as MSCs simultaneously receive the correct differentiation stimulus. This was confirmed through high production of ECM and adhesion and signalling molecules such as $\beta 1$-Integrin. Similar observations have been made in chondrocytes [15]. Interestingly, integrins have already been shown in several tissues to be able to mediate curcumin action [35,36]. As $\beta 1$-integrins are highly expressed in developing and adult cartilage, it is possible that the mechanism of action of curcumin in MSCs and chondrocytes might involve the $\beta 1$-integrin receptor signalling pathway.

Several studies have suggested that curcumin interacts with the TGF- $\beta$ signaling cascade, modulating the action of TGF- $\beta$. For example in renal cells curcumin blocks multiple sites of the TGF- $\beta$ signaling [37] or Smad inhibition in human proximal tubule cells [38]. However, no previously published in vitro studies have explored the potential interaction between TGF- $\beta$ and curcumin in chondrocytes.

In our experiments we did not observe inhibition (or a major difference in) the amounts of extracellular matrix production and signaling cascades when co-cultures were treated with the chondrogenic induction medium and curcumin or only with curcumin, we assume that here a possible interaction between curcumin and the TGF- $\beta$ signaling pathway does not have an inhibitory effect on positive chondrogenic signaling. However, interaction of curcumin and TGF- $\beta$ signaling in chondrocytes is an interesting point and will require further detailed investigations.

In the present study we demonstrated that a fairly low concentration of curcumin $(0.5 \mu \mathrm{M})$ was sufficient to 


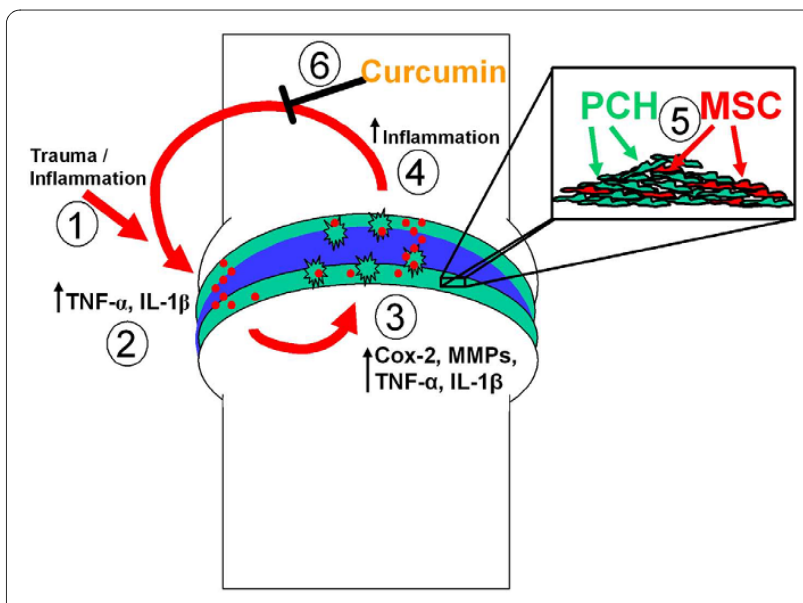

Figure 7 Schematic demonstrating the inflammatory cycle that inhibits chondrogenic differentiation of MSCs in OA and the effect of curcumin. Trauma, inflammation or a combination of both (1) lead to the production and accumulation of high levels of pro-inflammatory cytokines (2) in the joint. These trigger the production of additional pro-inflammatory cytokines which induce genes encoding prostaglandin synthesizing enzymes (that is, COX-2) and matrix degrading enzymes (3) such as MMPs and aggrecanases. These events promote cartilage degradation and stimulate further joint inflammation (4) and a self-perpetuating inflammatory and catabolic cascade develops. As illustrated, cartilage contains chondrocytes and MSC-like progenitors (5). Chemical or biological agents may help create a suitable microenvironment in order for these progenitor cells to undergo chondrogenesis and regenerate new cartilage in $\mathrm{OA}$. In this study we tested the hypothesis that phytochemical modulators of NF-KB can counteract this inflammatory and catabolic cascade and demonstrated that curcumin (6) has the capacity to block the action of pro-inflammatory cytokines in the joint thus disrupting the inflammatory cycle.

inhibit IL-1 $\beta$ induced activation of degradative pathways in MSCs. It must be noted that in this study we worked with low concentrations of curcumin, the highest concentration used being $5 \mu \mathrm{M}$. We chose this concentration based on previous studies in our laboratory demonstrating that canine MSCs do not tolerate curcumin concentrations higher than $5 \mu \mathrm{M}$. A recent study by Kim and coworkers demonstrated that chick MSCs treated with 20 $\mu \mathrm{M}$ curcumin become apoptotic and do not undergo chondrogenesis [39]. This clearly demonstrates that MSCs can only tolerate lower concentrations of curcumin in contrast to chondrocytes [40]. However, in vivo administered doses of curcumin in clinical trials differ greatly from this and have ranged between 2 to $10 \mathrm{~g}$ per day [4143]. An explanation for these high concentrations is that intestinal absorption of curcumin is fairly low, mainly due to the fact that curcumin is practically insoluble in water, and that it has a low bio-availability $[44,45]$. Despite its low bio-availability, efficacy has been demonstrated in several in vivo studies [46-48].

In order to develop a therapeutic strategy for OA/RA treatment with curcumin it would be interesting to deter- mine bioavailability of curcumin in the synovial fluid following various routes of administration (that is, oral, intra articular or topical). This is especially interesting as it may be also possible that in vivo curcumin exerts its effects via another organ (that is, the liver), which then leads to positive anabolic signalling in the joint. Therefore, to integrate our in vitro data with results from other studies, it is important to design and perform further in vivo studies. Furthermore, our experiments were carried out using canine derived cells and it may not be possible to make generalised statements about the activity of curcumin on MSC-like cells in other species.

\section{Conclusions}

Our results suggest that curcumin, the naturally occurring NF- $\kappa B$ inhibitor, protects MSCs from the deleterious effects of pro-inflammatory cytokines and thus creates a suitable microenvironment for MSCs to undergo chondrogenesis and this strategy may help stimulate cartilage regeneration in vitro (Figure 7). We therefore propose curcumin as a potential new therapeutic for the prophylactic treatment of OA/RA and for OA/RA cases where cartilage damage is marginal.

\begin{abstract}
Abbreviations
AP303a: alkaline phosphatase linked sheep anti-mouse; AP304A: sheep antirabbit secondary antibodies; DMSO: dimethylsulfoxide; COX-2: cyclooxygenase-2; CSPG: cartilage specific proteoglycans; ECM: extracellular matrix; ERK 1/ 2: extracellular regulated kinases 1 and 2; FCS: fetal calf serum; IKK: IKB kinase; IL: interleukin; MAB1965: anti- $\beta 1$-integrin antibody; MAB2015: monoclonal antiadult cartilage-specific proteoglycan antibody; MAPK: mitogen-activated protein kinase; MMP: matrix metalloproteinase; MSC: mesenchymal stem cell; MTT: 3-(4,5-dimethylthiazol-2-yl)-2,5-diphenyltetrazolium bromide; NF: nuclear factor; NF-KB: nuclear factor-KB; OA: osteoarthritis; PAB746: polyclonal anti-collagen type II antibody; PARP: (poly(ADP-Ribose) polymerase); PBS: phosphate buffered saline; RA: rheumatoid arthritis; Shc: src homology collagen; TNF: tumor necrosis factor.
\end{abstract}

\section{Competing interests}

The authors declare that they have no competing interests.

\section{Authors' contributions}

$\mathrm{CB}$ carried out the experimental work, data collection and interpretation, and manuscript preparation. AM, UM and MS conceived of the study design and coordinated the studies, data interpretation and manuscript preparation. All authors have read and approved the final manuscript.

\section{Acknowledgements}

Ms. Christina Pfaff and Ms. Ursula Schwikowski are gratefully acknowledged for their excellent technical assistance.

\section{Author Details}

'Musculoskeletal Research Group, Institute of Anatomy, Ludwig-MaximiliansUniversity Munich, Pettenkoferstrasse 11, D-80336 Munich, Germany, ${ }^{2}$ Division of Veterinary Medicine, School of Veterinary Medicine and Science, Faculty of Medicine and Health Sciences, University of Nottingham, Sutton Bonington Campus, Sutton Bonington LE12 5RD, UK and ${ }^{3}$ Clinic of Veterinary Surgery, Ludwig-Maximilians-University Munich, Veterinärstr. 13, 80539 Munich, Germany

Received: 3 February 2010 Revised: 14 May 2010

Accepted: 1 July 2010 Published: 1 July 2010 


\section{References}

1. Sandell $L$, Aigner T: Articular cartilage and changes in arthritis. An introduction: cell biology of osteoarthritis. Arthritis Res 2001, 3:107-113.

2. Elders MJ: The increasing impact of arthritis on public health. $J$ Rheumatol Supp/ 2000, 60:6-8.

3. Hunziker EB: Articular cartilage repair: are the intrinsic biological constraints undermining this process insuperable? Osteoarthritis Cartilage 1999, 7:15-28.

4. Buckwalter JA, Brown TD: Joint injury, repair, and remodeling: roles in post-traumatic osteoarthritis. Clin Orthop Relat Res 2004:7-16.

5. Heraud F, Heraud A, Harmand MF: Apoptosis in normal and osteoarthritic human articular cartilage. Ann Rheum Dis 2000, 59:959-965.

6. Todhunter PG, Kincaid SA, Todhunter RJ, Kammermann JR, Johnstone B, Baird AN, Hanson RR, Wright JM, Lin HC, Purohit RC: Immunohistochemical analysis of an equine model of synovitisinduced arthritis. Am J Vet Res 1996, 57:1080-1093.

7. Aigner T, Kim HA: Apoptosis and cellular vitality: issues in osteoarthritic cartilage degeneration. Arthritis Rheum 2002, 46:1986-1996.

8. Ding GJ, Fischer PA, Boltz RC, Schmidt JA, Colaianne JJ, Gough A, Rubin RA Miller DK: Characterization and quantitation of NF-kappaB nuclear translocation induced by interleukin-1 and tumor necrosis factoralpha. Development and use of a high capacity fluorescence cytometric system. J Biol Chem 1998, 273:28897-28905.

9. Shakibaei M, John T, Schulze-Tanzil G, Lehmann I, Mobasheri A: Suppression of NF-kappaB activation by curcumin leads to inhibition of expression of cyclo-oxygenase- 2 and matrix metalloproteinase- 9 in human articular chondrocytes: Implications for the treatment of osteoarthritis. Biochem Pharmacol 2007, 73:1434-1445.

10. Alsalameh S, Amin R, Gemba T, Lotz M: Identification of mesenchymal progenitor cells in normal and osteoarthritic human articular cartilage. Arthritis Rheum 2004, 50:1522-1532.

11. Jurenka JS: Anti-inflammatory properties of curcumin, a major constituent of Curcuma longa: a review of preclinical and clinical research. Altern Med Rev 2009, 14:141-153.

12. Aggarwal BB, Kumar A, Bharti AC: Anticancer potential of curcumin: preclinical and clinical studies. Anticancer Res 2003, 23:363-398.

13. Bharti AC, Donato N, Singh S, Aggarwal BB: Curcumin (diferuloylmethane) down-regulates the constitutive activation of nuclear factor-kappa B and IkappaBalpha kinase in human multiple myeloma cells, leading to suppression of proliferation and induction of apoptosis. Blood 2003, 101:1053-1062.

14. Schulze-Tanzil G, Mobasheri A, Sendzik J, John T, Shakibaei M: Effects of curcumin (diferuloylmethane) on nuclear factor kappaB signaling in interleukin-1 beta-stimulated chondrocytes. Ann N Y Acad Sci 2004, 1030:578-586.

15. Shakibaei M, Schulze-Tanzil G, John T, Mobasheri A: Curcumin protects human chondrocytes from IL-I1 beta-induced inhibition of collagen type II and beta1-integrin expression and activation of caspase-3: an immunomorphological study. Ann Anat 2005, 187:487-497.

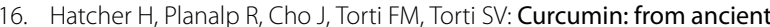
medicine to current clinical trials. Cell Mol Life Sci 2008, 65:1631-1652.

17. Mukhopadhyay A, Bueso-Ramos C, Chatterjee D, Pantazis P, Aggarwal BB: Curcumin downregulates cell survival mechanisms in human prostate cancer cell lines. Oncogene 2001, 20:7597-7609.

18. Lin JK, Pan MH, Lin-Shiau SY: Recent studies on the biofunctions and biotransformations of curcumin. Biofactors 2000, 13:153-158.

19. Conget PA, Minguell JJ: Phenotypical and functional properties of human bone marrow mesenchymal progenitor cells. J Cell Physio/ 1999, 181:67-73.

20. Jaiswal N, Haynesworth SE, Caplan Al, Bruder SP: Osteogenic differentiation of purified, culture-expanded human mesenchymal stem cells in vitro. J Cell Biochem 1997, 64:295-312.

21. Dominici M, Le Blanc K, Mueller I, Slaper-Cortenbach I, Marini F, Krause D, Deans R, Keating A, Prockop D, Horwitz E: Minimal criteria for defining multipotent mesenchymal stromal cells. The International Society for Cellular Therapy position statement. Cytotherapy 2006, 8:315-317.

22. Csaki C, Matis U, Mobasheri A, Ye H, Shakibaei M: Chondrogenesis, osteogenesis and adipogenesis of canine mesenchymal stem cells: a biochemical, morphological and ultrastructural study. Histochem Cell Biol $2007,128: 507-520$.
23. Shakibaei M, Schroter-Kermani C, Merker HJ: Matrix changes during long-term cultivation of cartilage (organoid or high-density cultures). Histol Histopathol 1993, 8:463-470.

24. Pittenger MF, Mackay AM, Beck SC, Jaiswal RK, Douglas R, Mosca JD, Moorman MA, Simonetti DW, Craig S, Marshak DR: Multilineage potential of adult human mesenchymal stem cells. Science 1999, 284:143-147.

25. Shakibaei M, De Souza P, Merker HJ: Integrin expression and collagen type II implicated in maintenance of chondrocyte shape in monolayer culture: an immunomorphological study. Cell Biol Int 1997, 21:115-125.

26. Csaki C, Keshishzadeh N, Fischer K, Shakibaei M: Regulation of inflammation signalling by resveratrol in human chondrocytes in vitro. Biochem Pharmacol 2008, 75:677-687.

27. Shakibaei M, Csaki C, Nebrich S, Mobasheri A: Resveratrol suppresses interleukin-1 beta-induced inflammatory signaling and apoptosis in human articular chondrocytes: potential for use as a novel nutraceutical for the treatment of osteoarthritis. Biochem Pharmacol 2008, 76:1426-1439

28. Csaki C, Mobasheri A, Shakibaei M: Synergistic chondroprotective effects of curcumin and resveratrol in human articular chondrocytes: inhibition of interleukin-1 beta-induced nuclear factor-kappaBmediated inflammation and apoptosis. Arthritis Res Ther 2009, 11:R165

29. Fernandes JC, Martel-Pelletier J, Pelletier JP: The role of cytokines in osteoarthritis pathophysiology. Biorheology 2002, 39:237-246.

30. Feldmann M, Brennan FM, Maini RN: Role of cytokines in rheumatoid arthritis. Annu Rev Immunol 1996, 14:397-440.

31. Gerlag DM, Ransone L, Tak PP, Han Z, Palanki M, Barbosa MS, Boyle D, Manning AM, Firestein GS: The effect of a T cell-specific NF-kappa B inhibitor on in vitro cytokine production and collagen-induced arthritis. J Immuno/ 2000, 165:1652-1658.

32. Miagkov AV, Kovalenko DV, Brown CE, Didsbury JR, Cogswell JP, Stimpson SA, Baldwin AS, Makarov SS: NF-kappaB activation provides the potential link between inflammation and hyperplasia in the arthritic joint. Proc Natl Acad Sci USA 1998, 95:13859-13864.

33. Park C, Moon DO, Choi IW, Choi BT, Nam TJ, Rhu CH, Kwon TK, Lee WH, Kim GY, Choi YH: Curcumin induces apoptosis and inhibits prostaglandin $\mathrm{E}(2)$ production in synovial fibroblasts of patients with rheumatoid arthritis. Int J Mol Med 2007, 20:365-372

34. Lev-Ari S, Strier L, Kazanov D, Elkayam O, Lichtenberg D, Caspi D, Arber N: Curcumin synergistically potentiates the growth-inhibitory and proapoptotic effects of celecoxib in osteoarthritis synovial adherent cells. Rheumatology (Oxford) 2006, 45:171-177.

35. Binion DG, Heidemann J, Li MS, Nelson VM, Otterson MF, Rafiee P: Vascular cell adhesion molecule-1 expression in human intestinal microvascular endothelial cells is regulated by PI 3-kinase/Akt/MAPK/ NF-kappaB: inhibitory role of curcumin. Am J Physiol Gastrointest Liver Physiol 2009, 297:G259-268.

36. Kim HI, Huang H, Cheepala S, Huang S, Chung J: Curcumin inhibition of integrin (alpha6beta4)-dependent breast cancer cell motility and invasion. Cancer Prev Res (Phila Pa) 2008, 1:385-391.

37. Gaedeke J, Noble NA, Border WA: Curcumin blocks multiple sites of the TGF-beta signaling cascade in renal cells. Kidney Int 2004, 66:112-120.

38. Hu Y, Liang H, Du Y, Zhu Y, Wang $X$ : Curcumin inhibits transforming growth factor-beta activity via inhibition of Smad signaling in HK-2 cells. Am J Nephrol 2010, 31:332-341

39. Kim DK, Kim SJ, Kang SS, Jin EJ: Curcumin inhibits cellular condensation and alters microfilament organization during chondrogenic differentiation of limb bud mesenchymal cells. Exp Mol Med 2009, 41:656-664

40. Clutterbuck A, Harris P, Mobasheri A: Comment on: comparison between chondroprotective effects of glucosamine, curcumin and diacerein in IL-1 beta-stimulated C-28/I2 chondrocytes. Osteoarthritis Cartilage 2009, 17:135-136. author reply 137.

41. Lao CD, Ruffin MTt, Normolle D, Heath DD, Murray SI, Bailey JM, Boggs ME, Crowell J, Rock CL, Brenner DE: Dose escalation of a curcuminoid formulation. BMC Complement Altern Med 2006, 6:10.

42. Cheng AL, Hsu CH, Lin JK, Hsu MM, Ho YF, Shen TS, Ko JY, Lin JT, Lin BR, Ming-Shiang W, Yu HS, Jee SH, Chen GS, Chen TM, Chen CA, Lai MK, Pu YS, Pan MH, Wang YJ, Tsai CC, Hsieh CY: Phase I clinical trial of curcumin, a chemopreventive agent, in patients with high-risk or pre-malignant lesions. Anticancer Res 2001, 21:2895-2900.

43. Sharma RA, McLelland HR, Hill KA, Ireson CR, Euden SA, Manson MM Pirmohamed M, Marnett LJ, Gescher AJ, Steward WP: Pharmacodynamic 
and pharmacokinetic study of oral Curcuma extract in patients with colorectal cancer. Clin Cancer Res 2001, 7:1894-1900.

44. Kurien BT, Scofield RH: Increasing aqueous solubility of curcumin for improving bioavailability. Trends Pharmacol Sci 2009, 30:334-335. author reply 335 .

45. Wang YJ, Pan MH, Cheng AL, Lin LI, Ho YS, Hsieh CY, Lin JK: Stability of curcumin in buffer solutions and characterization of its degradation products. J Pharm Biomed Anal 1997, 15:1867-1876.

46. Lim GP, Chu T, Yang F, Beech W, Frautschy SA, Cole GM: The curry spice curcumin reduces oxidative damage and amyloid pathology in an Alzheimer transgenic mouse. J Neurosci 2001, 21:8370-8377.

47. Ram A, Das M, Ghosh B: Curcumin attenuates allergen-induced airway hyperresponsiveness in sensitized guinea pigs. Biol Pharm Bull 2003, 26:1021-1024

48. Reyes-Gordillo K, Segovia J, Shibayama M, Vergara P, Moreno MG, Muriel P: Curcumin protects against acute liver damage in the rat by inhibiting NF-kappaB, proinflammatory cytokines production and oxidative stress. Biochim Biophys Acta 2007, 1770:989-996.

doi: 10.1186/ar3065

Cite this article as: Buhrmann et al., Curcumin mediated suppression of nuclear factor-?B promotes chondrogenic differentiation of mesenchymal stem cells in a high-density co-culture microenvironment Arthritis Research \& Therapy 2010, 12:R127

Submit your next manuscript to BioMed Central and take full advantage of:

- Convenient online submission

- Thorough peer review

- No space constraints or color figure charges

- Immediate publication on acceptance

- Inclusion in PubMed, CAS, Scopus and Google Scholar

- Research which is freely available for redistribution

Submit your manuscript at www.biomedcentral.com/submit
C) Biomed Central 